\title{
Pricing Game of Online Display Advertisement Publishers
}

\author{
Md. Tanveer Ahmed Changhyun Kwon * \\ Department of Industrial and Systems Engineering \\ University at Buffalo, The State University of New York
}

\begin{abstract}
We consider online display advertisement publishers who maximize the revenue by optimal pricing in an oligopoly setting. Each publisher interacts with others though setting cost-perimpression $(\mathrm{CPM})$ that affects the demand for everyone. Using the pseudoconcavity of the objective function, we prove that a unique best response Nash Equilibrium exists for each publisher. We also consider the sensitivity of the publisher while other publishers changes their CPM. In both cases, the best response of the publisher depends entirely on her current best response CPM. We provide an algorithm for finding the equilibrium and illustrate by numerical examples.
\end{abstract}

Keywords: online display advertising; optimal pricing; Nash equilibrium; newsvendor problem

\section{Introduction}

With a growing number audience, online advertisement has become an important marketing tool. With more than $76 \%$ US households having Internet access in 2008, the Internet accounts for approximately $20 \%$ of overall media consumption (Marketing Charts, 2008). According to Interactive Advertising Bureau (2010), online advertising yearly revenue is now nearing $\$ 25$ billion with a double digit yearly growth. The share of Internet in media consumption is expected to be more than 25\% by 2011 (Marketing Charts, 2008).

Search and display are two major formats of online advertising having $83 \%$ share of the market (Interactive Advertising Bureau, 2010). Search advertising refers to the use of search engine result pages such as Google or Bing where user can requests search relevant to keyword(s). The search engine responds by displaying the advertisements along with the relevant results. As a consumer makes a search indicating interest, search has more potential in generating instantaneous revenue. Therefore, search is preferred in many cases and has the biggest share (45\%) of the market. Display advertisement intends on creating consumer awareness or achieve memory rather than instantaneous response. With technological innovation and creativity, display advertisement is gaining market share (Interactive Advertising Bureau, 2010). On the other hand, the share of search advertising is

\footnotetext{
${ }^{*}$ Corresponding author. Tel.: +1 716645 4705; Fax: +1 715645 3302. E-mail: chkwon@buffalo.edu; Address: 400 Bell Hall, University at Buffalo, Buffalo, NY 14260, U.S.A.
} 
predicted to start dropping after it reaches 50\% market share (Marketing Charts, 2008). Interactive Advertising Bureau (2010) reports that from 2009 to 2010, the market share for display increased for $34 \%$ to $36 \%$ resulting about $5 \%$ relative gain while the market share for search remains the same.

In this paper, we consider a publisher who hosts display advertisements only. In general, payper-view (PPV) pricing mechanism is used for display. Cost-per-impression (CPM) is the pricing unit for PPV advertisements where loading of a web-page containing intended advertisement at user request is considered an impression or view. Though CPM is counted in increments of thousands, we consider unit increment for simplicity. This paper considers a monopoly where the publisher is willing to optimize advertising revenue by setting the price based on both her own parameter settings and market demand as a function of her price. Next, an oligopoly is considered where publishers interact by setting prices that affect the demand for each other. In fact, the current online advertising market is an oligopoly. According to Interactive Advertising Bureau (2010), the top 50 companies control $90 \%$ of the market share while the top 25 control $82 \%$ and the top 10 control $70 \%$ of the market share.

Though the Internet was commercially introduced in late 1990's, Mangani (2004) is known to be the first to propose a framework of choosing between PPV and search based pay-per-click (PPC) pricing methods. He considered a quasi-competitive market where each publisher is too small to influence the market. Fjell (2009) revisited the same problem and found that depending on the relation between click-trough rate and ratio of PPV and PPC market price, either PPV or PPC should be chosen, not both. To refine this study further, Kwon (2009) studied a capacity allocation problem between PPV and PPC advertising and finds mixed contract might be optimal in some cases. Recently, there have been a number of studies on display advertisements. Fjell (2010) studied his previous problem under a imperfect competition where a web publisher is a price setter. He assumed that number of PPV advertisements affects the page-view and hence PPV has the power to control the market. The issue of web banner (a type of display advertisement) advertisement scheduling was addressed by Amiri and Menon (2006). A dynamic selection and allocation of display advertisement model has been studied by Roels and Fridgeirsdottir (2009). Araman and Fridgeirsdottir (2011) proposed an uniform capacity allocation mechanism. They found a pricing policy that is easy to implement. A case of impatient advertisers was studied by Fridgeirsdottir and Asadolahi (2007). This problem was formulated as a queuing model where advertising slots correspond to servers. Though there has been a good number game theoretic studies, Moon and Kwon (2010) first studied a bargaining game between publisher and advertiser with option pricing scheme. To the best knowledge of the authors, an oligopoly between publishers has not been studied yet. Hence, we propose a monopoly and later an oligopoly where the publishers compete through setting prices that affects the available exogenous demand for each of them.

In this paper, we begin with a review of traditional optimal contract problem (Section 2). The publisher's problem is monopoly settings is studied is Section 3. Section 4 proposes an oligopolistic game between the publishers. The existence of a unique Nash equilibrium in this game is proved 
and a variational inequality formulation is provided in Section 4.1. In Section 4.2, we study an oligopolistic game with linear demand as a special case. This is followed by Section 5 where we propose a method to solve the oligopoly. A numerical example is provided in Section 6. In Section 7 , we provide managerial insights on the results. Section 8 concludes the paper.

\section{Review of Optimal Contract Problem}

In display advertising, pay-per-view (PPV) is a popular pricing method. We consider price-setting contract problems for display online advertisements with PPV pricing scheme of risk-neutral publishers in both monopoly and oligopoly settings. Payment for PPV is dependent on cost-perimpression (CPM), hence the revenue of a publisher depends on her capacity on the number impressions she can handle.

When we consider a publisher's optimal contract problem, there are two types of display advertisements with PPV pricing: one is advertisements by direct contracts with the publisher, and the other is so-called network advertisements (Roels and Fridgeirsdottir, 2009). The former type, henceforth termed as PPV advertisements, specifies a number to display the advertisements within a certain time-frame. Network advertisements are sourced through third-parties or network agencies who makes the contact with the advertisers. These network advertisements are always available for a flat rate lower price. The publisher generally tends to go for network advertisements to utilize the unused capacity after displaying the PPV advertisements.

When the publisher makes a direct contract with an advertiser to display a PPV advertisement, she promises a certain number of displays. For some reasons, when the publisher cannot display the contracted number, she has two options. She may consider refund to the advertiser for un-displayed numbers, or she may carry them over to the next time period. In the former case, the unit refund would be greater than or equal to the marginal revenue collected at the time of contract. We call this direct penalty. In the latter case, the publisher may lose potential contracts because of the carried-over, which we call indirect penalty. Therefore, for any strategy the publisher may take, there is a penalty when the contracted number of displays is not fulfilled. However, for network advertisements, there is no such promise on the number of displays but its marginal revenue is much smaller than PPV advertisements.

As the direct or indirect penalty is an important component of the operation of online advertising systems, the number of page-views for the future time period is critical to the publisher. A pageview is defined as a request to load a single page on a visitor's screen. This number is definitely

subject to uncertainty, i.e., the publisher can only have a forecast on this value i.e., the cumulative probability function (cdf), $F(\cdot)$ and probability density function (pdf), $f(\cdot)$ are known. We use a random variable $X$ to denote the uncertain number of page-views during the time period of interest.

Let $v$ denote the aggregate number of displays to promise. The CPM and penalty are denoted by $p$ and $h$, respectively. Although CPM is the cost for a thousand impressions, we use $p$ for one 
impression, for simplicity. Then, with the page-view $X$, the revenue of the publisher is:

$$
R(v)=p v-h(v-X)^{+}+q(X-v)^{+}
$$

where $x^{+}=\max (x, 0)$ and $q$ is the publisher's revenue per page-view of network advertisement. When there are extra page-views after fulfilling all contracts, the publisher will use those extra capacities for network advertisement. It is reasonable to assume that $q \leq p \leq h$. The publisher would not have any incentive to prefer PPV advertisement if $q>p$. Also, the publisher would prefer unlimited PPV if $p>h$. When we have the distribution information of $X$, the optimization problem

$$
\max _{v} \mathbb{E}[R(v)]
$$

is a simple stochastic optimization problem. Its solution is easily obtained as:

$$
v^{*}=F^{-1}\left(\frac{p-q}{h-q}\right)
$$

Ahmed and Kwon (2010) has studied problem (2) and found that it has a similar structure to the newsvendor problem and in fact, they are equivalent. The main difference between the two problems is that demand information is uncertain in the newsvendor problem, while capacity (page-views) is uncertain in the optimal contract problem (2).

Consider a newsvendor who has to make decision everyday about the amount of paper $Q$ he would be ordering before start delivering the paper and observe the realized demand $D$. The purchase cost of paper is $c$ per paper and he sells them at a rate of $\pi$ per paper. The actual demand $D$ is an unknown a priori but characterized by cdf, $G($.$) and pdf, \mathrm{g}($.$) respectively. At$ the end of the sales, the excess papers are returned and he receives the salvage price $s$ per paper. We assume $\pi>c>s$, while $s$ can be negative. The cost of lost opportunity of selling is $l$ per unit. The optimum ordering quantity for the risk-neutral newsvendor can be derived as:

$$
Q^{*}=G^{-1}\left(\frac{\pi+l-c}{\pi+l-s}\right)
$$

With $p=\pi-c, h=\pi-s, q=-l$ and $X=D$, we find $Q^{*}=v^{*}$ and thus newsvendor problem is equivalent to publisher's problem (2). Closing this section, we introduce the following definitions for the rest of the paper:

Definition 1. A function $f$ from $K \subseteq \mathbb{R}$ into $\mathbb{R}$ is said to be

1. pseudoconcave if for all $x_{1}, x_{2} \in K$,

$$
\frac{\partial f\left(x_{1}\right)}{\partial x}\left(x_{2}-x_{1}\right) \leq 0 \quad \Longrightarrow \quad f\left(x_{2}\right) \leq f\left(x_{1}\right)
$$


2. strictly pseudoconcave if for all $x_{1}, x_{2} \in K$ when $x_{1} \neq x_{2}$,

$$
\frac{\partial f\left(x_{1}\right)}{\partial x}\left(x_{2}-x_{1}\right) \leq 0 \quad \Longrightarrow \quad f\left(x_{2}\right)<f\left(x_{1}\right)
$$

Definition 2. A mapping $F$ from $K \subseteq \mathbb{R}^{n}$ into $\mathbb{R}^{n}$ is said to be

1. pseudomonotone if for all $x, y \in K$,

$$
F(y)^{T}(x-y) \geq 0 \Longrightarrow F(x)^{T}(x-y) \geq 0
$$

2. strictly pseudomonotone if for all $x, y \in K$ when $x \neq y$,

$$
F(y)^{T}(x-y) \geq 0 \Longrightarrow F(x)^{T}(x-y)>0
$$

Definition 3. A mapping $F$ from $K \subseteq \mathbb{R}^{n}$ into $\mathbb{R}^{n}$ is Lipschitz continuous with modulus $L$ if there exists a constant $L \geq 0$ such that

$$
\|F(x)-F(y)\| \leq L\|x-y\|
$$

for all $x, y \in K$.

\section{Monopoly Setting: Stochastic Page-View and Deterministic De- mand}

Suppose $h$ and $q$ are fixed. For any given price $p>0$ and (random) page-view $X$, the revenue is:

$$
r(v, p, X)=p v-h(v-X)^{+}+q(X-v)^{+}
$$

where $x^{+}=\max (x, 0)$. The optimal contract size is:

$$
v(p)=\arg \max _{v \geq 0} \mathbb{E}_{X}[r(v, p, X)]=F^{-1}\left(\frac{p-q}{h-q}\right)
$$

where $F$ is the cumulative distribution function of $X$ and $F^{-1}$ is its inverse. Note that the optimal contract size in (5) does not depend on the demand which is impacted by the CPM set by the publisher. If the demand is greater than $v(p)$, the publisher should reject some portion of demand. If the demand is less than $v(p)$, the contract size is set to the demand size. As a result, the realized size of the contract and the resultant revenue depend on demand as well. We assume that the demand depends on the price and denote it by $d(p)$. To avoid trivial cases, we assume that $d(q)>v(q)$ and $v(h)>d(h)$. Hence, the realized contract size $m(p)$ is:

$$
m(p) \equiv \min (v(p), d(p))=\min \left(F^{-1}\left(\frac{p-q}{h-q}\right), d(p)\right)
$$


The expectation of the realized revenue is then:

$$
R(p)=\mathbb{E}[r(m(p), p, X)]
$$

We are interested in the following optimization problem:

$$
\max _{p \geq 0} R(p) \quad \text { subject to } \quad q \leq p \leq h
$$

where

$$
\begin{aligned}
R(p) & =p m(p)-h \mathbb{E}\left[(m(p)-X)^{+}\right]+q \mathbb{E}\left[(X-m(p))^{+}\right] \\
& =(p-q) m(p)+q \mu-(h-q) m(p) F(m(p))+(h-q) \int_{0}^{m(p)} x f(x) d x
\end{aligned}
$$

and $\mu=E[x]$. Note that $v(p)$ is an increasing function. We make the following assumptions on the demand function $d(p)$ :

Assumption 1. The demand function $d(p)$ satisfies following regularity properties:

1. $p$ is defined on $\Omega=[q, h]$.

2. $\lim _{p \rightarrow h} d(p)=\epsilon$, where $\epsilon$ is a small and economically meaningless value.

3. $d(p)$ is continuous, bounded, and twice continuously differentiable in $p$ on $\Omega$.

4. $d(p)$ is strictly decreasing; i.e., $\frac{\partial d(p)}{\partial p}<0$, on $\Omega$.

5. $d(p)$ is either convex, concave or both; i.e., $\frac{\partial d(p)}{\partial p}$ is monotone.

Properties 1, 2, and 3 are standard. As $p \rightarrow h, d(p)$ becomes infinitesimally small. This rules out the possibility that $p$ becomes infinite. The assumption that demand function is strictly decreasing (Property 4) refers to most general case of price elasticity of demand. Except two theoretical cases of Veblen good and Giffen good, this assumption is almost universal.

Figure 1 shows $v(p)$ for a gamma page-view distribution, logit demand $d(p)$ and the corresponding $m(p)$. The corresponding revenue function is shown in Figure 2.

Since $d(p)$ is strictly decreasing and $v(p)$ strictly increasing, $m(p)$ can be defined as:

$$
m(p)=\left\{\begin{array}{lll}
v(p) & \text { if } & q<p<\bar{p} \\
d(p) & \text { if } & \bar{p} \leq p
\end{array}\right.
$$

where $\bar{p}$ is the price such that $v(\bar{p})=d(\bar{p})$. Since $v(p)$ is strictly increasing and $d(p)$ is strictly decreasing, such $\bar{p}$ is unique. When $m(p)=v(p)$, the revenue function becomes:

$$
R^{v}(p)=(p-q) v(p)+q \mu-(h-q) v(p) F(v(p))+(h-q) \int_{0}^{v(p)} x f(x) d x
$$




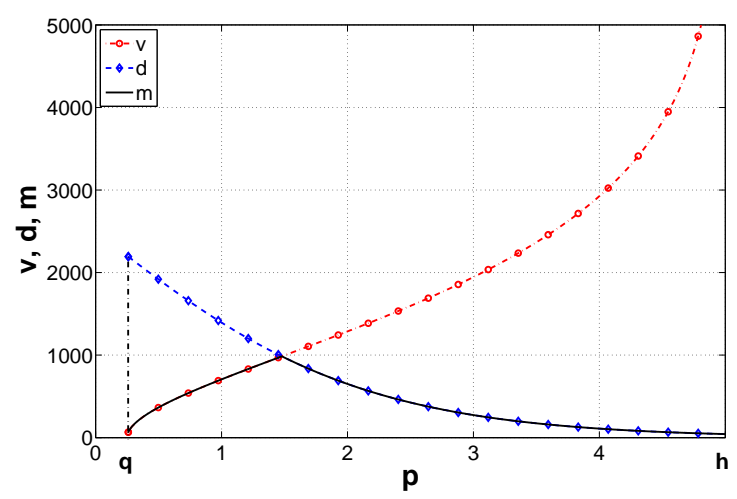

Figure 1: $v, d$, and $m$ as function of $p$

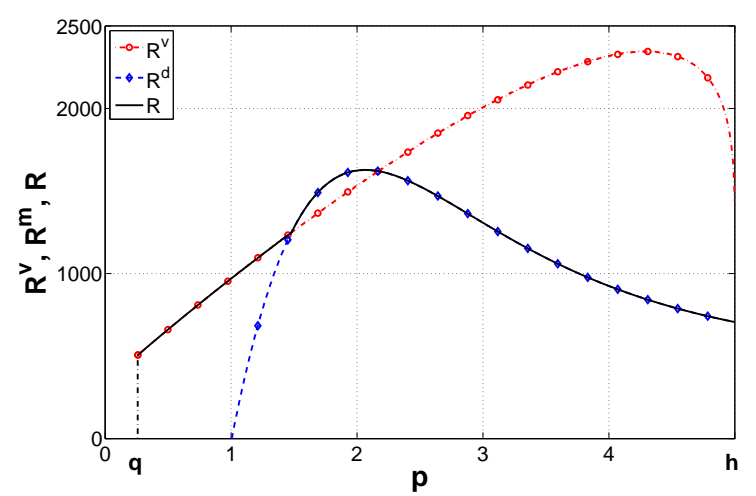

Figure 2: $R^{v}, R^{d}$, and $R$ as function of $p$

Similarly, when $m(p)=d(p)$, we obtain

$$
R^{d}(p)=(p-q) d(p)+q \mu-(h-q) d(p) F(d(p))+(h-q) \int_{0}^{d(p)} x f(x) d x
$$

Note that both $R^{v}(p)$ and $R^{d}(p)$ are continuous. Therefore the continuity of $R(p)$ is easily verified as $R^{v}(\bar{p})=R^{d}(\bar{p})$. We can also show that $R(p)$ is continuously differentiable (see Appendix).

We observe that a solution to the problem (6) always concurs with $R^{d}(p)$ :

Lemma 1. With increasing $v(p)$ and decreasing $d(p)$, the publisher needs to consider the exogenous demand only in setting the optimal price. That is

$$
p^{*} \equiv \arg \max R(p)=\arg \max R^{d}(p)
$$

In addition, we have $p^{*} \in[\bar{p}, h]$ where $\bar{p}$ is defined in (8).

Proof. The proof is found in Appendix. In the rest of this paper, all omitted proofs are also found in Appendix.

Lemma 1 implies that instead of the problem (6), we have the following publisher's problem:

$$
\max R^{d}(p)=(p-q) d(p)+q \mu-(h-q) d(p) F(d(p))+(h-q) \int_{0}^{d(p)} x f(x) d x
$$

subject to $p \in \Omega$

In the game model we will be considering later, the shape of $R^{d}$ is important.

Proposition 1. The revenue function $R^{d}(p)$ is strictly pseudoconcave for $p \in \Omega$ under Assumption 1.

Since $R^{d}(p)$ is strictly pseudoconcave, there exists a unique $p^{*} \in \Omega$. The optimality condition 
is provided in a variational-inequality form: $p^{*} \in \Omega$ is a solution if and only if

$$
\frac{\partial R^{d}\left(p^{*}\right)}{\partial p}\left(p-p^{*}\right) \leq 0 \quad \forall p \in \Omega
$$

This variational inequality form of the optimality condition will be used to formulate an equilibrium problem in an oligopoly of several publishers.

\section{Oligopolistic Pricing Game of Publishers}

We provide a formulation for an oligopolistic competition of publishers, where each publisher's demand depends on own price as well as other publishers' prices. While we maintain the same modeling framework as in the monopoly case considered in the previous section, we need to modify the demand function and the feasible set of each player accordingly.

Let us consider the publisher $i$ 's revenue of the form:

$$
r_{i}\left(v_{i}, p_{i}, X_{i}\right)=p_{i} v_{i}-h_{i}\left(v_{i}-X_{i}\right)^{+}+q_{i}\left(X_{i}-v_{i}\right)^{+}
$$

where the subscript $i$ denotes the publisher $i$ 's parameters and variables as those are already defined in the previous section. Without considering exogenous demand, the optimal contract size is as follows:

$$
v_{i}\left(p_{i}\right)=\arg \max _{v_{i} \geq 0} \mathbb{E}_{X_{i}}\left[r_{i}\left(v_{i}, p_{i}, X_{i}\right)\right]=F_{i}^{-1}\left(\frac{p_{i}-q_{i}}{h_{i}-q_{i}}\right)
$$

We assume that the demand of publisher $i$ depends on its own price $p_{i}$ and other publisher's prices as well. We denote the publisher $i$ 's demand by $d_{i}\left(p_{i}, p_{-i}\right)$, where $p_{-i}$ is the vector of prices of all publishers except publisher $i$. Since a publisher cannot make a contract whose size is greater than the demand, the realized contract size becomes

$$
m_{i}\left(p_{i}, p_{-i}\right)=\min \left(v_{i}\left(p_{i}\right), d_{i}\left(p_{i}, p_{-i}\right)\right)
$$

The realized revenue is then

$$
R_{i}\left(p_{i}, p_{-i}\right)=\mathbb{E}_{X_{i}}\left[r_{i}\left(m_{i}\left(p_{i}, p_{-i}\right), p_{i}, X_{i}\right)\right]
$$

We have already proved that a publisher needs to consider exogenous demand in maximizing her revenue. Denoting the optimal prices of other publishers by $p_{-i}^{*}$, we consider the following problem of each publisher $i$ (best response problem):

$$
\begin{aligned}
\max _{p_{i}} R_{i}\left(p_{i}, p_{-i}^{*}\right)=\max _{p_{i}} & R_{i}^{d}\left(p_{i}, p_{-i}^{*}\right)=\left(p_{i}-q_{i}\right) d_{i}\left(p_{i}, p_{-i}^{*}\right)+q_{i} \mu_{i} \\
& \quad-\left(h_{i}-q_{i}\right) d_{i}\left(p_{i}, p_{-i}^{*}\right) F_{i}\left(d_{i}\left(p_{i}, p_{-i}^{*}\right)\right)+\left(h_{i}-q_{i}\right) \int_{0}^{d_{i}\left(p_{i}, p_{-i}^{*}\right)} x f(x) d x
\end{aligned}
$$


subject to

$$
p_{i} \in \Omega_{i}\left(p_{-i}^{*}\right) \equiv\left\{p_{i}: q_{i} \leq p_{i} \leq h_{i}, \quad d_{i}\left(p_{i}, p_{-i}^{*}\right) \geq 0\right\}
$$

Note that the set of feasible price of publisher $i$ depends on the other publishers' optimal price $p_{-i}^{*}$. This leads to a generalized Nash equilibrium problem (Pang and Fukushima, 2005). However, with some mild assumptions on the demand function, we can provide a regular Nash equilibrium problem formulation; we will discuss this later in this section.

We make the following assumptions in consistent to Assumption 1:

Assumption 2. For any publisher $i, i=1, \ldots, n$, the demand $d_{i}\left(p_{i}, p_{-i}\right)$ satisfies the following regularity properties:

1. Each $p_{i}$ is defined on $\Omega_{i}=\left[q_{i}, h_{i}\right]$.

2. for all $p_{-i} \in \Omega_{i}, \lim _{p_{i} \rightarrow h_{i}} d_{i}\left(p_{i}, p_{-i}\right)=\epsilon$, where $\epsilon$ is a small and economically meaningless value. In other words, $\inf _{p_{i} \in \Omega_{i}} d_{i}\left(p_{i}, p_{-i}\right)=\epsilon$.

3. $d_{i}\left(p_{i}, p_{-i}\right)$ is continuous, bounded, and twice continuously differentiable on the strategy space $\Omega=\Omega_{1} \times \ldots \times \Omega_{n}$.

4. $\partial d_{i}\left(p_{i}, p_{-i}\right) / \partial p_{i}<0$.

5. $d_{i}\left(p_{i}, p_{-i}\right)$ is either convex or concave or both; i.e., $\partial d_{i}\left(p_{i}, p_{-i}\right) / \partial p_{i}$ is monotone.

6. $\partial d_{i}\left(p_{i}, p_{-i}\right) / \partial p_{j}>0$ for all $j \neq i$.

Succeeding the exposition of the previous section, we obtain the following result:

Lemma 2. Suppose Assumption 2 holds. For any given $p_{-i} \in \prod_{j=1, j \neq i}^{n} \Omega_{j}$, the revenue function $R_{i}\left(\cdot, p_{-i}\right)$ of publisher $i$ is continuously differentiable and strictly pseudoconcave on $\Omega_{i}$.

Let us define

$$
\begin{aligned}
p & =\left[p_{1}, p_{2}, \ldots, p_{n}\right]^{T} \\
H(p) & =\left[-\frac{\partial R_{1}^{d}(p)}{\partial p_{1}},-\frac{\partial R_{2}^{d}(p)}{\partial p_{2}}, \ldots,-\frac{\partial R_{n}^{d}(p)}{\partial p_{n}}\right]^{T}
\end{aligned}
$$

Then, it is easy to prove the pseudomonotonicity of $H$ following Theorem 3.1 of Karamardian (1976).

Lemma 3. When $R_{i}^{d}$ is a strictly pseudoconcave function of $p_{i}$, the mapping $H(\cdot)$ is strictly pseudomonotone on $\prod_{i=1}^{n} \Omega_{i}$.

Since $\Omega$ is non-empty and compact, a solution of variational inequality problem with the mapping (15) exists (Crouzeix, 1997). In Section 5, we formulate the variational inequality as a fixedpoint problem and the apply extragradient method to solve (15) using Lemma 3.

In Sections 4.1 and 4.2, we will formulate variational inequality for the equilibrium problems. 


\subsection{Nash Equilibrium Problem for Strictly Positive Demand}

In this section, we consider demand functions that satisfy the following assumption, in addition to Assumption 2:

Assumption 3. Each publisher's demand is strictly positive at $p_{i}=h_{i}$ for all cases, i.e., $d_{i}\left(h_{i}, p_{-i}\right)>$ 0 for all $p_{-i} \in \prod_{j=1, j \neq i}^{n} \Omega_{j}$.

With this assumption, each publisher's feasible set is reduced to the set $\Omega_{i}=\left\{p_{i}: q_{i} \leq\right.$ $\left.p_{i} \leq h_{i}\right\}$ which is independent of $p_{-i}$; therefore the corresponding equilibrium problem becomes a regular Nash equilibrium problem, which is easily solved under the assumptions made in this paper. Examples of such demand functions include the following popular functions:

- Multinomial Logit Demand: $d_{i}\left(p_{i}, p_{-i}\right)=D \frac{\exp \left(-\lambda_{i} p_{i}\right)}{\sum_{j=1}^{n} \exp \left(-\lambda_{j} p_{j}\right)}$

- Exponential Demand: $d_{i}\left(p_{i}, p_{-i}\right)=\exp \left(a_{i}-b_{i i} p_{i}+\sum_{j=1, j \neq i}^{n} b_{i j} p_{j}\right)$

- Constant-Elasticity Demand: $d_{i}\left(p_{i}, p_{-i}\right)=a_{i} p_{1}^{-b_{i 1}} p_{2}^{b_{i 2}} \cdots p_{i}^{b_{i i}} \cdots p_{n}^{b_{i n}}$

The linear demand function is discussed in the next section, as it does not satisfy Assumption 3.

Under Assumption 3, the best response problem of publisher $i$ is as follows:

$$
\begin{aligned}
\max _{p_{i}} R_{i}^{d}\left(p_{i}, p_{-i}^{*}\right)=\left(p_{i}-q_{i}\right) & d_{i}\left(p_{i}, p_{-i}^{*}\right)+q_{i} \mu_{i} \\
& \quad-\left(h_{i}-q_{i}\right) d_{i}\left(p_{i}, p_{-i}^{*}\right) F_{i}\left(d_{i}\left(p_{i}, p_{-i}^{*}\right)\right)+\left(h_{i}-q_{i}\right) \int_{0}^{d_{i}\left(p_{i}, p_{-i}^{*}\right)} x f(x) d x
\end{aligned}
$$

subject to

$$
p_{i} \in \Omega_{i}=\left\{p_{i}: q_{i} \leq p_{i} \leq h_{i}\right\}
$$

With the strict pseudoconcavity of $R_{i}^{d}\left(\cdot, p_{-i}\right)$, we may write its optimality condition as the following variational inequality problem: to find $p_{i} \in \Omega_{i}$ such that

$$
\frac{\partial R_{i}^{d}\left(p_{i}^{*}, p_{-i}^{*}\right)}{\partial p_{i}}\left(p_{i}-p_{i}^{*}\right) \leq 0 \quad \forall p_{i} \in \Omega_{i}
$$

Concatenating for all publishers, we obtain the following variational inequality formulation for the equilibrium problem: to find $p^{*} \in \Omega$ such that

$$
H\left(p^{*}\right)^{T}\left(p-p^{*}\right) \geq 0 \quad \forall p \in \Omega
$$


where

$$
\begin{aligned}
p & =\left[p_{1}, p_{2}, \ldots, p_{n}\right]^{T} \\
H(p) & =\left[-\frac{\partial R_{1}^{d}(p)}{\partial p_{1}},-\frac{\partial R_{2}^{d}(p)}{\partial p_{2}}, \ldots,-\frac{\partial R_{n}^{d}(p)}{\partial p_{n}}\right]^{T} \\
\Omega & =\prod_{i=1}^{n} \Omega_{i}
\end{aligned}
$$

The problem (20) is a $n$-dimensional variational inequality problem with a strictly pseudomonotone operator and a nonempty, convex and compact feasible set. This class of problems has a welldeveloped literature; see Facchinei and Pang (2003) and Harker and Pang (1990). We want to assure that the equilibrium described by (20) exists. We provide the following result:

Proposition 2 (Existence and Uniqueness of Nash Equilibrium). Under Assumptions 2 and 3, there exists a solution to the variational inequality problem (20); hence a Nash equilibrium exists. Further, since $H$ is strictly pseudomonotone, it is unique.

Proof. The set $\Omega$ obviously is a nonempty, compact and convex subset of $\mathbb{R}^{n}$ and $H$ is a continuous mapping from $\Omega$ into $\mathbb{R}^{n}$. Therefore applying Browder's fixed-point theorem (Browder, 1968), the existence of Nash Equilibrium is proved. The uniqueness of the solution when $F$ is strictly pseudomonotone is assured by Lemma 3.2 of Yao (1994).

The algorithm for obtaining the equilibrium will be discussed in Section 5 .

\subsection{Nash Equilibrium Problem with Linear Demand}

In this section, we consider linear demand functions. It is apparent that linear demand functions does not satisfy Assumption 3; therefore we cannot directly obtain a regular Nash equilibrium problem. We consider the set

$$
\Omega_{i}\left(p_{-i}\right) \equiv\left\{p_{i}: q_{i} \leq p_{i} \leq h_{i}, d_{i}\left(p_{i}, p_{-i}\right) \geq 0\right\}
$$

where $\Omega_{i}$ depends on others' prices $p_{-i}$. This general consideration will generally lead to a generalized Nash game and the corresponding quasi-variational inequality of the form: to find $p^{*} \in \Omega\left(p^{*}\right)$ such that

$$
H\left(p^{*}\right)^{T}\left(p-p^{*}\right) \geq 0 \quad \forall p \in \Omega\left(p^{*}\right)
$$

where

$$
\Omega\left(p^{*}\right)=\prod_{i=1}^{n} \Omega_{i}\left(p_{-i}^{*}\right)
$$

Quasi-variational inequalities (QVI) are relatively less studied than variational inequalities and generally harder to solve (Pang and Fukushima, 2005). However, in this section, we will show that 
the pricing game can be described by a variational inequality, not a quasi-variational inequality.

We make the following assumption of linear demand:

Assumption 4. Each demand function $d_{i}\left(p_{i}, p_{-i}\right)$ is linear in $p_{i}$ and each component of $p_{-i}$, i.e., it is of the form $d_{i}\left(p_{i}, p_{-i}\right)=a_{i}-b_{i i} p_{i}+\sum_{j=1, j \neq i}^{n} b_{i j} p_{j}$.

With this assumption, the set of feasible prices is

$$
\Omega_{i}\left(p_{-i}\right)=\left\{p_{i}: q_{i} \leq p_{i} \leq h_{i}, \quad a_{i}-b_{i i} p_{i}-\sum_{j=1, j \neq i}^{n} b_{i j} p_{j} \geq 0\right\}
$$

For the linear demand case, we provide the following lemma:

Lemma 4. Under Assumption 4, for all $p_{-i}$, the unconstrained solution of publisher $i$ is not bounded by the nonnegativity constraint of demand. That is,

$$
q_{i} \leq p_{i, \text { unconstrained }}^{*} \leq p_{i}^{\max }\left(p_{-i}\right)
$$

where $p_{i, \text { unconstrained }}^{*}=\arg \max _{p_{i}} R_{i}^{d}\left(p_{i}, p_{-i}\right)$ and $p_{i}^{\max }$ is the smallest price at which $d_{i}\left(p_{i}^{\max }, p_{-i}\right)=$ 0 .

Lemma 4 indicates that we need to consider only the basic interval constraint $q_{i} \leq p_{i} \leq h_{i}$, i.e.,

$$
\Omega_{i}=\left\{q_{i} \leq p_{i} \leq h_{i}\right\}
$$

Consequently, the equilibrium problem is described by the following variational inequality:

$$
H\left(p^{*}\right)^{T}\left(p-p^{*}\right) \geq 0 \quad \forall p \in \Omega
$$

Then we obtain the existence and uniqueness of the solution to (26).

Proposition 3 (Existence and Uniqueness of Nash Equilibrium). Under Assumptions 2 and 4 , there exists a unique solution to the variational inequality problem (26); hence a Nash equilibrium exist.

From Propositions 2 and 3, the equilibrium exists and is unique under both Assumptions 3 and 4. In Section 5, we discuss how we obtain the equilibrium.

\subsection{Sensitivity Study}

We are now interested in the best response of player $i$, when other players' strategies and own set of feasible actions change. We first consider the case when the best response of player $i$ is strictly within the boundary, i.e., $p_{i}^{*} \in\left(q_{i}, h_{i}\right)$. In this case, $p_{i}^{*}$ must satisfy the following optimality condition:

$$
\left.\frac{\partial R^{d}\left(p_{i}, p_{-i}\right)}{\partial p_{i}}\right|_{p_{i}=p_{i}^{*}}=d_{i}\left(p_{i}^{*}, p_{-i}\right)+\left.\left\{\left(p_{i}^{*}-q_{i}\right)-\left(h_{i}-q_{i}\right) F\left[d_{i}\left(p_{i}^{*}, p_{-i}\right)\right]\right\} \frac{\partial d_{i}\left(p_{i}, p_{-i}\right)}{\partial p_{i}}\right|_{p_{i}=p_{i}^{*}}=0
$$


Let us define $G\left(p_{i}^{*}, p_{-i}\right)=\left(p_{i}^{*}-q_{i}\right)-\left(h_{i}-q_{i}\right) F\left[d_{i}\left(p_{i}^{*}, p_{-i}\right)\right]$. We observe that $G\left(p_{i}^{*}, p_{-i}\right) \geq 0$ and $\left.\frac{\partial^{2} d_{i}\left(p_{i}, p_{-i}\right)}{\partial p_{i} \partial p_{j}}\right|_{p_{i}=p_{i}^{*}} \leq 0$. Using implicit differentiations, we obtain the following results from (27):

$$
\begin{aligned}
& \left.\frac{\partial p_{i}}{\partial q_{i}}\right|_{p_{i}=p_{i}^{*}}=1-F\left[d_{i}\left(p_{i}^{*}, p_{-i}\right)\right] \geq 0 \\
& \left.\frac{\partial p_{i}}{\partial h_{i}}\right|_{p_{i}=p_{i}^{*}}=F\left[d_{i}\left(p_{i}^{*}, p_{-i}\right)\right] \geq 0
\end{aligned}
$$

and

$$
\begin{array}{r}
\left.\frac{\partial p_{i}}{\partial p_{j \neq i}}\right|_{p_{i}=p_{i}^{*}}=\frac{1}{d_{i}\left(p_{i}^{*}, p_{-i}\right)}\left[\left.\left\{G\left(p_{i}^{*}, p_{-i}\right)+\left(h_{i}-q_{i}\right) f\left[d_{i}\left(p_{i}^{*}, p_{-i}\right)\right] d_{i}\left(p_{i}^{*}, p_{-i}\right)\right\} \frac{\partial d_{i}\left(p_{i}, p_{-i}\right)}{\partial p_{j}}\right|_{p_{i}=p_{i}^{*}}\right. \\
\left.-\left.\frac{\partial^{2} d_{i}\left(p_{i}, p_{-i}\right)}{\partial p_{i} \partial p_{j}}\right|_{p_{i}=p_{i}^{*}} G^{2}\left(p_{i}^{*}, p_{-i}\right)\right] \geq 0
\end{array}
$$

From (28), we note that $p_{i}^{*}$ may increase when $q_{i}$ increases. However, the rate of change of $p_{i}^{*}$ is comparatively less than $q_{i}$ as the right-hand-side of (28) is less than 1 . Similarly, we find that $p_{i}^{*}$ may increase when $h_{i}$ increases though at a comparatively slower rate than $h_{i}$ as equation (29) suggests. From equation (30), we find that $p_{i}^{*}$ would increase as $p_{j}$ would increase. However, simplifying the rate of change of $p_{i}^{*}$ may be analytically difficult.

Now we consider the cases when the best response player $i$ is at the boundary, i.e., $p_{i}^{*}=q_{i}$ or $p_{i}^{*}=h_{i}$. For $p_{i}^{*}=q_{i}$, we obtain the following lemma:

Lemma 5. If the best response of player $i$ is such that $p_{i}^{*}=q_{i}$, we obtain the following result:

$$
\left.\frac{\partial R_{i}^{d}\left(p_{i}, p_{-i}^{*}\right)}{\partial p_{i}}\right|_{p_{i}=q_{i}}=0
$$

Also, in this case we have $p_{i}^{*}=q_{i}=\bar{p}_{i}$ and $m_{i}\left(p_{i}^{*}\right)=v\left(\bar{p}_{i}\right)=d_{i}\left(\bar{p}_{i}, p_{-i}^{*}\right)=0$, where $\bar{p}_{i}$ is defined as similar as in (8)

While $q_{i}$ is a bound for feasible $p_{i}$, this lemma indicates that the first-order optimality condition (27) always holds when $p_{i}^{*}=q_{i}$; therefore, the same sensitivity results as before also apply.

We consider the effect of change of $p_{j \neq i}$ while $p_{i}^{*}=q_{i}$. As $p_{j \neq i}$ increases, $d_{i}\left(p_{i}, p_{-i}\right)$ also increases from 0 . To respond to other publishers' change in strategies, the publisher will have the same demand for a CPM, $\tilde{p}_{i}>\bar{p}_{i}=q_{i}$. This would result in $v\left(\tilde{p}_{i}\right)>d_{i}\left(\tilde{p}_{i}, p_{-i}^{*}\right)$ i.e. $m_{i}\left(p_{i}^{*}\right)=d_{i}\left(\tilde{p}_{i}, p_{-i}^{*}\right)$. As a result, increasing her own CPM, $p_{i}$ will be the best response to the increase of other publishers' CPM.

We provide the following lemma for the case when $p_{i}^{*}=h_{i}$. 
Lemma 6. If the best response of player is such that $p_{i}^{*}=h_{i}$, we obtain the following result:

$$
\left.\frac{\partial R_{i}^{d}\left(p_{i}, p_{-i}^{*}\right)}{\partial p_{i}}\right|_{p_{i}=h_{i}} \geq 0
$$

We now consider the effect of change of $p_{j \neq i}$ while $p_{i}^{*}=h_{i}$. As $p_{j \neq i}$ decreases $d_{i}\left(p_{i}, p_{-i}\right)$ also decreases. However, as long as $\left.\frac{\partial R_{i}^{d}\left(p_{i}, p_{-i}^{*}\right)}{\partial p_{i}}\right|_{p_{i}=h_{i}}>0$, the best response of player $i$ would remain same, i.e. $p_{i}^{*}=h_{i}$. If $p_{j \neq i}$ decreases in way such that $\left.\frac{\partial R_{i}^{d}\left(p_{i}, p_{-i}^{*}\right)}{\partial p_{i}}\right|_{p_{i}=h_{i}}=0$, further decrease would result in decrease of $d_{i}\left(p_{i}^{*}, p_{i}\right)$. In this case, as we find in equation (30), player $i$ 's best response $\mathrm{CPM}, p_{i}^{*}$ decreases.

\section{$5 \quad$ Fixed-Point Reformulation and Extragradient Method}

In both cases, we have a finite-dimensional variational inequality problem with the pseudomonotone operator mapping. While there are many methods are available for monotone problems in the literature, there are limited numbers of methods for pseudomonotone problems. In this section, we consider the Extragradient Method (Facchinei and Pang, 2003).

Most methods for solving variational inequality problems rely on the fixed-point problem reformulation of the variational inequality. The variational inequality problem (20) is equivalent to the following fixed-point problem:

$$
p=P_{\Omega}[p-\alpha H(p)]
$$

where $P_{\Omega}[\cdot]$ is the projector on $\Omega$ with a constant $\alpha>0$. In particular, $P_{\Omega}[x]$ is the solution of the following minimum norm projection problem:

$$
\min _{y \in \Omega}(y-x)^{T}(y-x)
$$

Note that (34) is a convex quadratic minimization problem, which is usually easily solved. Based on (33), the following is the basic projection algorithm (or fixed-point algorithm):

$$
p^{k+1}=P_{\Omega}\left[p^{k}-\alpha H\left(p^{k}\right)\right]
$$

at each iteration $k$. While the convergence of this scheme for monotone problems is assured, we need an advanced method for solving pseudomonotone problems. The iterative scheme of the Extragradient Method is:

$$
p^{k+1}=P_{\Omega}\left[p^{k}-\alpha H\left(P_{\Omega}\left[p^{k}-\alpha H\left(p^{k}\right)\right]\right)\right]
$$


or

$$
\begin{aligned}
q^{k+1} & =P_{\Omega}\left[p^{k}-\alpha H\left(p^{k}\right)\right] \\
p^{k+1} & =P_{\Omega}\left[p^{k}-\alpha H\left(q^{k+1}\right)\right]
\end{aligned}
$$

where we take an extra step in each iteration. The convergence is assured by the following result.

Proposition 4 (Convergence). Suppose the mapping $H$ is Lipschitz continuous on $\Omega$ with modulus L. The Extragradient Method converges to a solution of the variational inequality problem (20) for all $\alpha \in(0,1 / L)$.

Proof. Since $H$ is pseudomonotone and $\Omega$ is a closed convex set, the convergence of the Extragradient Method is assured by Theorem 12.1.11 of Facchinei and Pang (2003).

\section{$6 \quad$ Numerical Results}

In this section, we use a numerical example of an asymmetric oligopoly to gain certain insights about the equilibrium. We use the popular logit demand model:

$$
D_{i}(p)=D \frac{\exp \left(-a_{i} p_{i}\right)}{1+\sum_{j=1}^{n} \exp \left(-a_{j} p_{j}\right)}, \quad \text { where } \quad a_{i}>0, a_{j}>0
$$

We consider an oligopoly where the publishers are asymmetric. The total market demand is assumed to be $D=20000$. The page-view is considered identical and follows gamma distribution with shape factor $\alpha=2$ and scale factor $\beta=1000$. To find different possible scenarios, we consider five set of asymmetric publishers with different set of parameters chosen randomly such that $q_{i} \in[0.25,1.25]$, $h_{i} \in[1.5,5]$, and $a_{i} \in[0.5,1.5]$ for all publishers. Figure 3 shows the effect of number of publishers $(n)$ on optimal CPM of player $1\left(p_{1}^{*}\right)$. We find that $p_{1}^{*}$ decreases as the number of competitors $n$ increases. The demand for publisher $1\left(d_{1}\left(p_{1}^{*}, p_{-1}^{*}\right)\right)$, in a similar manner, always decreases as $n$ increases (Figure 4). Similar to demand, Figure 5 shows that the revenue for publisher 1 $\left(R_{1}\left(p_{1}^{*}, p_{-1}^{*}\right)\right)$ decreases as $n$ increases. As $p_{1}^{*}$ as well as $d_{1}\left(p_{1}^{*}, p_{-1}^{*}\right)$ decreases with the increase of $n$, the total market revenue increases with a decreasing rate with increasing number of publishers (Figure 6).

We consider a game of five asymmetric publishers to find the effect of change of strategy space (network CPM, $q_{1}$ and the penalty, $\left.h_{1}\right)$ of publisher 1 . For logit demand $(D=5000)$ and identical gamma distribution of page-view with shape factor $\alpha=2$ and scale factor $\beta=1000$, we consider the change of the penalty $\left(h_{1}\right)$ first. The parameters are chosen in similar manner mentioned above. We consider network CPM, for publisher $1, q_{1}=0.25$. In this case, we find two scenarios. First, as seen in Figure 7, $p_{1}^{*}$ decreases as $h_{1}$ decreases from the highest value, at a slower rate than $h_{1}$ as we have found in (29). With further decrease of $h_{1}$, eventually we find a point $h_{1}^{\alpha}$ such that $p_{1}^{*}=h_{1}$. This relation, $p_{1}^{*}=h_{i}$ remains same for any $h_{1} \leq h_{1}^{\alpha}$. In the second case (Figure 8), we also have a critical point $h_{1}^{\alpha}$ such that for any $h_{1} \leq h_{1}^{\alpha}$, we have $p_{1}^{*}=h_{1}$. However, if $h_{1}$ very high $\left(h_{1}>h_{1}^{\alpha}\right)$, 
we have $p_{1}^{*}=q_{1}$. This indicates that when $h_{1}$ is sufficiently small, the publisher will set $p_{1}$ equal to $h_{1}$ as she loses very little as penalty. However, for large values of $h_{1}$, the publisher should either set $p_{1}^{*}=h_{1}$ or $p_{1}^{*}=q_{1}$. In case $p_{1}^{*}=h_{1}$, the publisher does not have any actual penalty. She simply loses the potential revenue. When $p_{1}^{*}=q_{1}$, the possible volume of penalty becomes too high and the publisher is rather safe to go for network advertisement only. We also study the effect of change of network CPM, $q_{1}$ for same gamma distribution. In this case, we consider penalty, for publisher $1, h_{1}=5$. Figure 9 shows the effect of change of $q_{1}$ on $p_{1}^{*}$. As suggested by equation (28), this figure shows that $p_{1}^{*}$ increases at a slower rate than $q_{1}$ for $q_{1} \leq q_{1}^{\alpha}$. For $q_{1}>q_{1}^{\alpha}$, if $p_{1}^{*}$ reaches $h_{1}$ while $q_{1}=q_{1}^{\alpha}, p_{1}^{*}$ remains equal to $h_{1}$ for $q_{1}>q_{1}^{\alpha}$.

We consider an asymmetric game of two publishers to study the effect of change of best response strategy of one publisher as the other changes his CPM. We consider logit demand model again $(D=2000)$ and identical gamma distribution. Again, the parameters are chosen at random such that $q_{i} \in[0.25,1.25], h_{i} \in[3,5]$, and $a_{i} \in[0.5,1.5]$ for both the publishers. We find that (as equation (30) suggests) one publisher increases her best response CPM as the other publisher increases her CPM (Figure 10). Note that even though the other publisher makes a large change of the CPM, the range of best response for a publisher is quite small i.e. the level of sensitivity is very low.

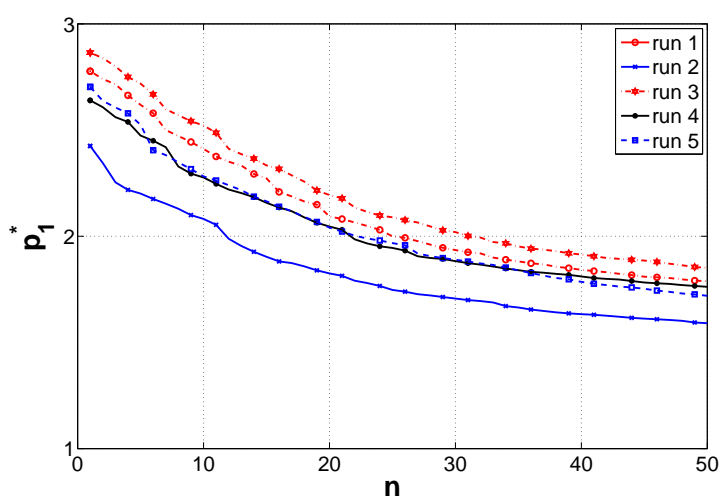

Figure 3: Optimal CPM of publisher $1, p_{1}^{*}$ as the number of publishers changes

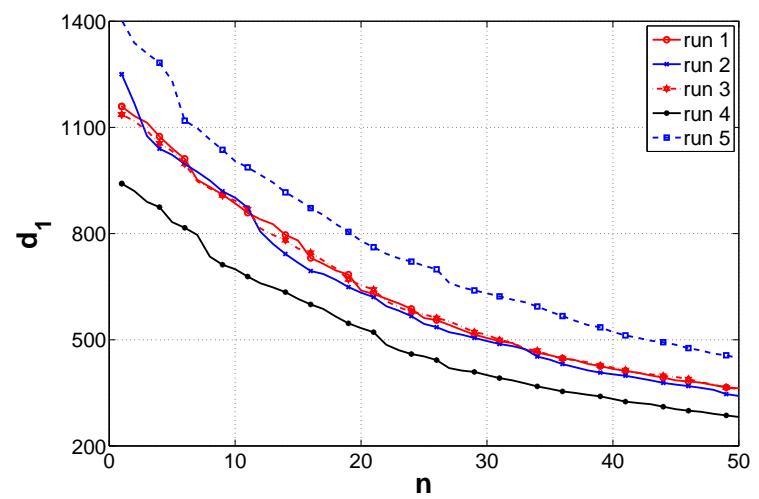

Figure 4: Change of demand of publisher 1, $d_{1}\left(p_{1}^{*}, p_{-1}^{*}\right)$ as the number of publishers changes

\section{$7 \quad$ Managerial Insight}

As we have seen in Section 6 (Figures 3-6), a publisher's response to the level of competition is somewhat consistent with what we observe in other markets where players tend to lower prices in response to more competition. The optimal pricing for a publisher decreases as the level of competition increases. Each new competitor affects demand of a publisher differently, and depending on demand variations, a publisher may decide to make a small decrease in her CPM or a significant 


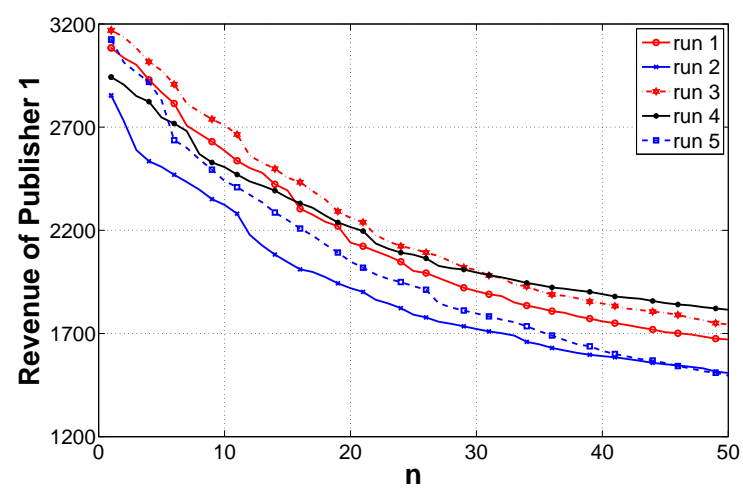

Figure 5: Revenue for publisher $1, R_{1}\left(p_{1}^{*}, p_{-1}^{*}\right)$ as the number of publishers changes

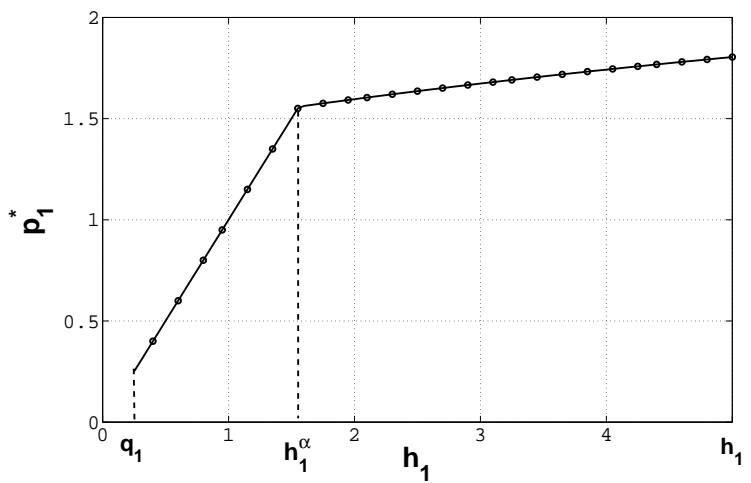

Figure 7: Optimal CPM, $p_{1}^{*}$ for publisher 1 as penalty, $h_{1}$ changes (Case 1 )

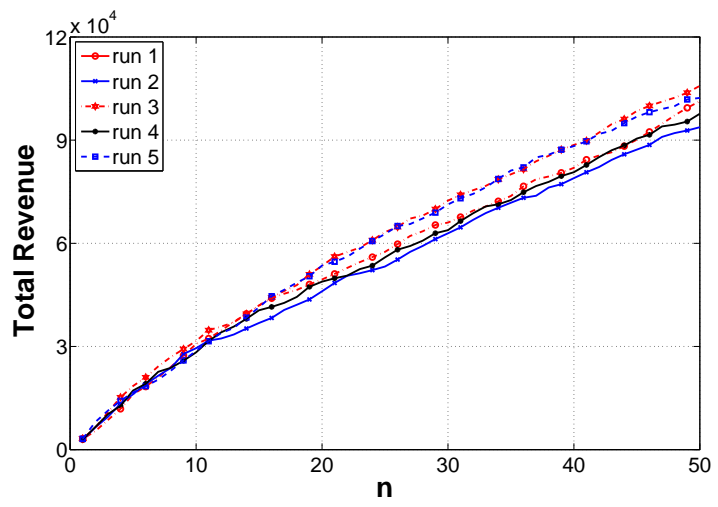

Figure 6: Total revenue as the number of publishers changes

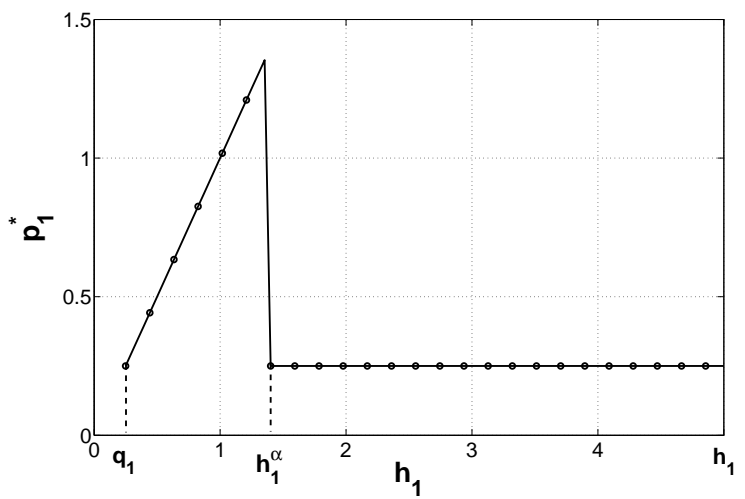

Figure 8: Optimal CPM, $p_{1}^{*}$ for publisher 1 as penalty, $h_{1}$ changes (Case 2 )

pricing adjustment.

In terms of pricing in the face of uncertainty, we observe (Figure 11) that as the variance increases, the publisher increases price. With increased variance in page-view, the risk increases. Each publisher responds to the increased risk by increasing own price. This is also common in other markets.

However, the publisher's optimal response to changes of the penalty parameter is not straightforward and not comparable to other markets (Figures 7-8). It is because the penalty is specific to the online advertisement context. As the penalty increases, the publisher has two completely different courses of actions, either to increase CPM proportionally or to choose network advertisement only. The publisher's sensitivity to other publisher's change of CPM is very low (Figure 10). Any unilateral change in CPM affects the total demand as well as respective demand of all the publishers. However, responding to this change in demand by changing CPM may not be always optimal for a publisher. An increase in price may result in reduction of total demand and may even decrease own demand resulting lower expected revenue. On the other hand, the net increase 


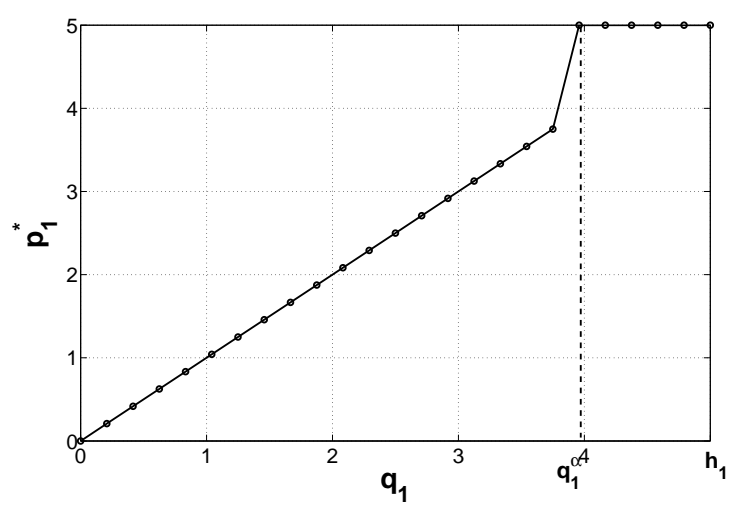

Figure 9: Optimal CPM, $p_{1}^{*}$ as network CPM, $q_{1}$ changes

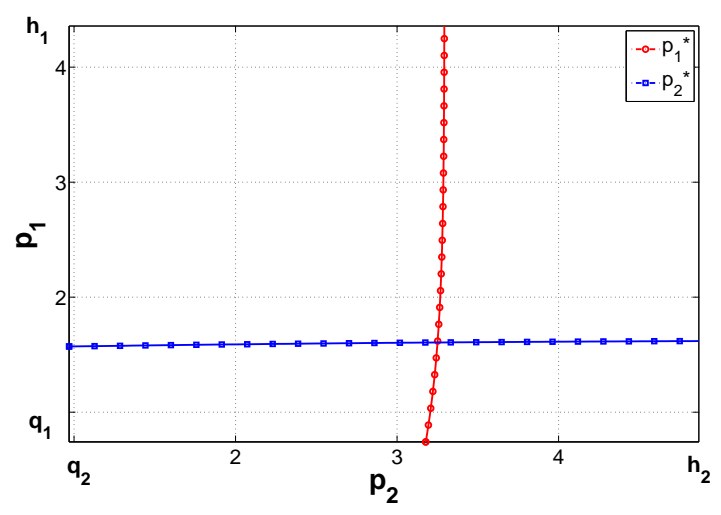

Figure 10: Optimal CPM of one publisher as the other publisher changes strategy (CPM)

of own demand due to lowered CPM may result in lower expected revenue.

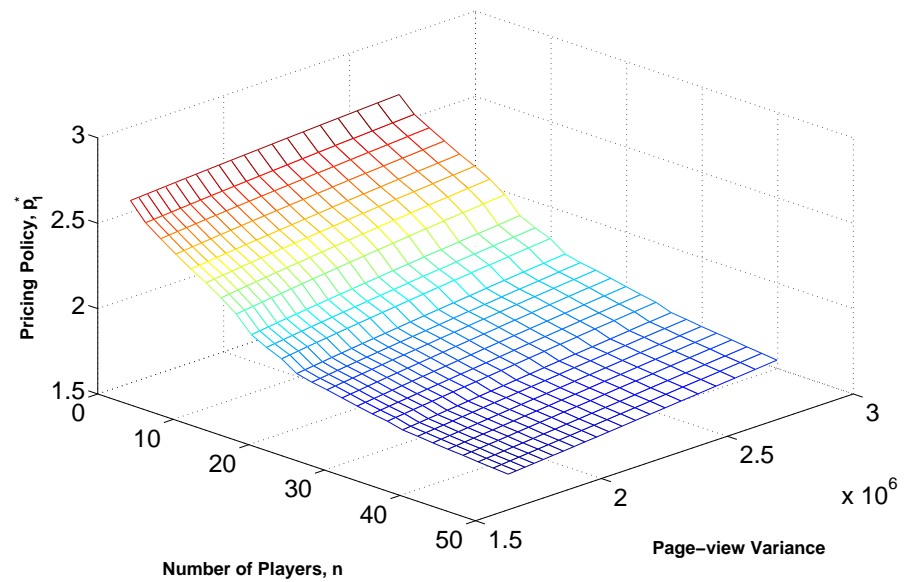

Figure 11: Optimal pricing for different level of competition and uncertainty (variance)

\section{Conclusion}

In online display advertisement, revenue is generated through impression based pricing which uses pay-per-view (PPV) with cost-per-impression (CPM) as pricing basis. Accordingly, CPM is an important consideration for maximizing revenue. Both page-view capacity as well as market demand determine the actual contract size and the resultant revenue. Here, we consider a publisher who wants to maximize her revenue by setting the CPM. She also needs to consider the corresponding market demand and suggested contract size based on her pricing range and page-view distribution. The optimal size for the publisher in this case is the minimum of the two mentioned above.

The publisher's problem has been studied in both monopoly and oligopoly settings. In monopoly 
setting, the publisher's expected revenue function is found to be strictly pseudo-concave resulting in an unique revenue maximizing CPM. We also find that the revenue maximizing CPM lies in a region where the market demand is less than or equal to the suggested contract size. The market demand decreases and suggested contract size increases as the CPM increases. For every suggested contract size for a CPM, there is an exactly same market demand at a higher CPM resulting in more revenue. Therefore, in setting optimal CPM, the publisher needs to consider the market demand only.

In the oligopolistic setting, we consider a game where every publisher considers her own strategy space as well as the CPMs set by the other publishers. In this game, the publishers interact by setting individual CPMs that determine the available demand for each of the publishers. As the number of publishers increases, the optimal CPM of each publisher decreases as each responds to lower demand resulting from more competition. Accordingly, revenue for each publisher decreases as the number of competitors increases.

In general, in both monopoly and oligopoly settings, the optimal CPM increases as either network CPM or penalty rate for unfulfilled display increases. However, these results are not general. As network CPM increases, optimal CPM increases first and will eventually reach penalty rate and would remain there as network CPM increases. Here, the publisher would go for PPV advertisement if CPM is equal to the penalty rate and she gets revenue exactly for what she could show and does not incur any penalty. While penalty rate increases, the optimal CPM increases and remains exactly same as penalty rate. However, there exists a value of penalty rate above which the CPM either increases at a slower rate or becomes equal to network CPM. In the last case, the penalty is very high and the publisher should display network advertisement only.

The sensitivity of a publisher's best response in oligopoly while other publishers are changing respective CPM depends not only on others' behavior but also on her own strategy space. If optimal CPM is strictly within the strategy space i.e., between network CPM and penalty rate, each publisher should increase price while the other publishers increase the CPM. If the publisher's optimal CPM is found to be equal penalty rate, the best response of the publisher remains insensitive for a while as other publishers are decreasing the CPM. As the other publishers are decreasing their CPM, the demand for publisher remains so high that she could still charge the same CPM. However, if the other publishers decrease their CPM sufficiently, then the demand will be considerably low and she has to decrease the CPM as appropriate response. On the other hand, if optimal CPM is equal to network CPM, she increases CPM as the network CPM increases though at a slower rate.

In this paper, we find that a publisher needs to consider market demand exclusively in choosing CPM and subsequent contact decision. This result is valid for both monopoly as well as oligopoly settings. Future research direction could include the consideration of search based advertising, more specifically click-through rate (CTR) and corresponding pay-per-click (PPC). In such a scenario, the publisher might be willing to balance between this two types of advertising format considering the demand based on pricing of them. 


\section{References}

Ahmed, M. T. and C. Kwon (2010). Optimal Contract Problems in Online Advertising with Risk Considerations. Working paper.

Amiri, A. and S. Menon (2006). Scheduling web banner advertisements with multiple display frequencies. Systems, Man and Cybernetics, Part A: Systems and Humans, IEEE Transactions on $36(2), 245-251$.

Araman, V. and K. Fridgeirsdottir (2011). A Uniform Allocation Mechanism and Cost-perImpression Pricing for Online Advertising. Working paper.

Browder, F. (1968). The Fixed Point Theory of Multi-valued Mappings in Topological Vector Spaces. Mathematische Annalen 177(4), 283-301.

Cambini, A. and L. Martein (2009). Generalized convexity and optimization: theory and applications. Number 616. Springer Verlag.

Crouzeix, J. (1997). Pseudomonotone variational inequality problems: existence of solutions. Mathematical Programming 78(3), 305-314.

Facchinei, F. and J. Pang (2003). Finite-dimensional Variational Inequalities and Complementarity Problems. Springer Verlag.

Fjell, K. (2009). Online advertising: Pay-per-view versus pay-per-click: A comment. Journal of Revenue and Pricing Management 2(3), 200-206.

Fjell, K. (2010). Online advertising: Pay-per-view versus pay-per-click with market power. Journal of Revenue 8 Pricing Management 9(3), 198-203.

Fridgeirsdottir, K. and S. Asadolahi (2007). Revenue management for online advertising: Impatient advertisers. Technical report, Working paper, London Business School.

Harker, P. and J. Pang (1990). Finite-dimensional Variational Inequality and Nonlinear Complementarity Problems: A Survey of Theory, Algorithms and Applications. Mathematical Programming $48(1), 161-220$.

Interactive Advertising Bureau (2010, October 12). Internet Ad Revenues Break Records, Climb to More Than $\$ 12$ Billion for First Half of 10. http://www.iab.net/about_the_iab/recent_ press_releases/press_release_archive/press_release/pr-101210. Online; accessed 10July-2010.

Karamardian, S. (1976). Complementarity Problems over Cones with Monotone and Pseudomonotone Maps. Journal of Optimization Theory and Applications 18(4), 445-454. 
Kwon, C. (2009). Single-period balancing of pay-per-click and pay-per-view online display advertisements. Journal of Revenue $\&$ Pricing Management.

Mangani, A. (2004). Online advertising: Pay-per-view versus pay-per-click. Journal of Revenue and Pricing Management 2(4), 295-302.

Marketing Charts (2008, January 22). US Online Advertising Market to Reach $\$ 50 \mathrm{~B}$ in 2011. urlhttp://www.marketingcharts.com/interactive/us-online-advertising-market-to-reach50b-in-2011-3128/. Online; accessed 10-February-2011.

Moon, Y. and C. Kwon (2010). Online advertisement service pricing and an option contract. Electronic Commerce Research and Applications.

Pang, J. and M. Fukushima (2005). Quasi-variational Inequalities, Generalized Nash Equilibria, and Multi-leader-follower Games. Computational Management Science 2(1), 21-56.

Roels, G. and K. Fridgeirsdottir (2009). Dynamic revenue management for online display advertising. Journal of Revenue \& Pricing Management 8(5), 452-466.

Yao, J. (1994). Variational Inequalities with Generalized Monotone Operators. Mathematics of Operations Research 19(3), 691-705.

\section{Appendix}

\section{Proof of Lemma 1}

Proof. From (8), the revenue function could be defined as:

$$
R(p)=\left\{\begin{array}{lll}
R^{v}(p) & \text { if } \quad q<p<\bar{p} \\
R^{d}(p) & \text { if } \quad \bar{p} \leq p
\end{array}\right.
$$

As $v(p)$ is increasing and $d(p)$ is decreasing with $\mathrm{p}$, there are prices $q \leq p_{1} \leq \bar{p} \leq p_{2}$ such that $v\left(p_{1}\right)=d\left(p_{2}\right)$. Also, we find that

$$
\begin{aligned}
& R^{d}\left(p_{2}\right)-R^{v}\left(p_{1}\right)=\left(p_{2}-p_{1}\right) d\left(p_{2}\right) \geq 0 \\
& R^{d}\left(p_{2}\right) \geq R^{v}\left(p_{1}\right)
\end{aligned}
$$

Hence we prove the lemma.

\section{Proof of Proposition 1}

We first prove the following lemma: 
Lemma A.1. A continuously differentiable function $f$ from $K \subseteq \mathbb{R}$ into $\mathbb{R}$ is strictly pseudoconcave if $f$ is strictly increasing and then strictly decreasing.

Proof. Let $\left.\frac{\partial f(x)}{\partial x}\right|_{x=\hat{x}}=0$. Then we have

$$
\begin{aligned}
& \frac{\partial f(x)}{\partial x}>0 \text { if } x<\hat{x} \\
& \frac{\partial f(x)}{\partial x}<0 \text { if } x>\hat{x}
\end{aligned}
$$

Pick $x_{1}, x_{2} \in K$ and $x_{1} \neq x_{2}$. We consider four cases:

(i) $x_{1}<\hat{x}$ and $x_{2}<\hat{x}$ : We have

$$
\left.\frac{\partial f(x)}{\partial x}\right|_{x=x_{1}}\left(x_{2}-x_{1}\right) \leq 0 \Longrightarrow x_{2}-x_{1} \leq 0 \Longrightarrow f\left(x_{2}\right)<f\left(x_{1}\right)
$$

(ii) $x_{1}<\hat{x}$ and $x_{2} \geq \hat{x}$ : We have

$$
\left.\frac{\partial f(x)}{\partial x}\right|_{x=x_{1}}\left(x_{2}-x_{1}\right) \leq 0 \Longrightarrow x_{2}-x_{1} \leq 0 \Longrightarrow \text { Contradiction }
$$

(iii) $x_{1} \geq \hat{x}$ and $x_{2}<\hat{x}$ : We have

$$
\left.\frac{\partial f(x)}{\partial x}\right|_{x=x_{1}}\left(x_{2}-x_{1}\right) \leq 0 \Longrightarrow x_{2}-x_{1} \geq 0 \Longrightarrow \text { Contradiction }
$$

(iv) $x_{1} \geq \hat{x}$ and $x_{2} \geq \hat{x}$ : We have

$$
\left.\frac{\partial f(x)}{\partial x}\right|_{x=x_{1}}\left(x_{2}-x_{1}\right) \leq 0 \Longrightarrow x_{2}-x_{1} \geq 0 \Longrightarrow f\left(x_{2}\right)<f\left(x_{1}\right)
$$

Therefore in all cases, we have $\left.\frac{\partial f(x)}{\partial x}\right|_{x=x_{1}}\left(x_{2}-x_{1}\right) \leq 0$ implies $f\left(x_{2}\right)<f\left(x_{1}\right)$.

We now show that $R(p)$ is continuously differentiable. The first order derivative of function (7) is

$$
\frac{\partial R(p)}{\partial p}=m(p)+\frac{\partial m(p)}{\partial p}[(p-q)-(h-q) F(m(p))]
$$

Suppose $m(p)=v(p)$, i.e., $v(p)<d(p)$. We have then

$$
\frac{\partial R^{v}(p)}{\partial p}=v(p) \geq 0
$$

since $v(p)=F^{-1}\left(\frac{p-q}{h-q}\right)$. In addition, $R^{v}(p)$ is concave as its second order derivative is negative. Therefore $R^{v}(p)$ is a monotonically increasing concave function. Suppose $m(p)=d(p)$, i.e., $v(p)>$ 
$d(p)$. We have then

$$
\frac{\partial R^{d}(p)}{\partial p}=d(p)+\frac{\partial d(p)}{\partial p}[(p-q)-(h-q) F(d(p))]
$$

The continuous differentiability of $R(p)$ can be verified by examining the expected revenue at $\bar{p}$. From (A.2) and (A.3), we can see $R(p)$ is differentiable at $\bar{p}$, since

$$
\left.\frac{\partial R^{d}(p)}{\partial p}\right|_{p=\bar{p}}=d(\bar{p})=v(\bar{p})=\left.\frac{\partial R^{d}(p)}{\partial p}\right|_{p=\bar{p}}
$$

Let us define

$$
G(p)=(p-q)-(h-q) F(d(p))
$$

At $p=\bar{p}$, we have

$$
G(p)=(p-q)-(h-q) F(v(p))=(p-q)-(h-q) \frac{p-q}{h-q}=0
$$

When $p<\bar{p}$, we have

$$
\begin{aligned}
d(p) & >v(p) \\
F(d(p)) & >F(v(p)) \\
F(d(p)) & >\frac{p-q}{h-q} \\
G(p) & <0
\end{aligned}
$$

Similarly, for $p>\bar{p}, G(p)>0$.Also, we find that

$$
\frac{\partial G(p)}{\partial p}=1-(h-q) f(d(p)) \frac{\partial d(p)}{\partial p} \geq 0 \quad \forall p \geq 0
$$

We make the following observations:

Lemma A.2. The revenue function $R^{d}(p)$ for $p \in(q, \bar{p}]$ is strictly increasing.

Proof of Lemma A.2. Since $d(p)>0, \frac{\partial d(p)}{\partial p}<0$ and $G(p) \leq 0$ for $p \leq \bar{p}$ we have

$$
\frac{\partial R^{d}(p)}{\partial p}=d(p)+\frac{\partial d(p)}{\partial p} G(p)>0
$$

Lemma A.3. If the demand function $d(p)$ is concave, the revenue function $R^{d}(p)$ for $p \in(\bar{p}, h]$ is strictly increasing and then strictly decreasing.

Proof of Lemma A.3. Since we have $\frac{\partial d(p)}{\partial p}<0, \frac{\partial^{2} d(p)}{\partial^{2} p} \leq 0, G(p)>0$ and $\frac{\partial G(p)}{\partial p}>0$, the revenue 
function $R^{d}(p)$ is concave as:

$$
\frac{\partial^{2} R^{d}(p)}{\partial p^{2}}=\frac{\partial d(p)}{\partial p}+\frac{\partial^{2} d(p)}{\partial^{2} p} G(p)+\frac{\partial d(p)}{\partial p} \frac{\partial G(p)}{\partial p}<0
$$

In addition we note

$$
\left.\frac{\partial R^{d}(p)}{\partial p}\right|_{p=\bar{p}}=d(\bar{p})>0
$$

and

$$
\left.\frac{\partial R^{d}(p)}{\partial p}\right|_{p=h}=\left.\frac{\partial d(p)}{\partial p}\right|_{p=h} G(h)<0
$$

Therefore we obtain the lemma.

Lemma A.4. If the demand function $d(p)$ is convex, the revenue function $R^{d}(p)$ for $p \in(\bar{p}, h]$ is strictly increasing and then strictly decreasing.

Proof of Lemma A.4. From the convexity of $d(p)$, we have $\frac{\partial^{2} d(p)}{\partial^{2} p} \geq 0$. For any $\hat{p}, \tilde{p} \in(\bar{p}, h]$, we have

$$
\begin{aligned}
{[d(\hat{p})-d(\tilde{p})](\hat{p}-\tilde{p}) } & <0 \\
{[G(\hat{p})-G(\tilde{p})](\hat{p}-\tilde{p}) } & >0 \\
{\left[\frac{\partial d(\hat{p})}{\partial p}-\frac{\partial d(\tilde{p})}{\partial p}\right](\hat{p}-\tilde{p}) } & \geq 0
\end{aligned}
$$

since we have $\frac{\partial d(p)}{\partial p}<0, \frac{\partial G(p)}{\partial p}>0$ and $\frac{\partial^{2} d(p)}{\partial^{2} p} \geq 0$, respectively, for all $\in(\bar{p}, h]$.

We observe $\frac{\partial R^{d}}{\partial p}(\bar{p})>0$ and $\frac{\partial R^{d}}{\partial p}(h)<0$, therefore there must exist a price $\hat{p} \in(\bar{p}, h]$ such that $\frac{\partial R^{d}}{\partial p}(\hat{p})=0$. If we can show that such a point is unique, then we obtain the desired result.

Suppose there exists $\tilde{p} \in(\bar{p}, h]$ such that $\frac{\partial R^{d}}{\partial p}(\tilde{p})=0$ and $\hat{p} \neq \tilde{p}$. That is we have

$$
\begin{aligned}
& d(\hat{p})+\frac{\partial d(\hat{p})}{\partial p} G(\hat{p})=0 \\
& d(\tilde{p})+\frac{\partial d(\tilde{p})}{\partial p} G(\tilde{p})=0
\end{aligned}
$$

Then, we must have

$$
d(\hat{p})-d(\tilde{p})+\frac{\partial d(\hat{p})}{\partial p} G(\hat{p})-\frac{\partial d(\tilde{p})}{\partial p} G(\tilde{p})=0
$$

However, from (A.8) and the facts that $\frac{\partial d(\hat{p})}{\partial p}<0$ and $G(\tilde{p})>0$, we have

$$
[d(\hat{p})-d(\tilde{p})](\hat{p}-\tilde{p})+\frac{\partial d(\hat{p})}{\partial p}[G(\hat{p})-G(\tilde{p})](\hat{p}-\tilde{p})+G(\tilde{p})\left[\frac{\partial d(\hat{p})}{\partial p}-\frac{\partial d(\tilde{p})}{\partial p}\right](\hat{p}-\tilde{p})<0
$$

In consequence

$$
\left\{d(\hat{p})-d(\tilde{p})+\frac{\partial d(\hat{p})}{\partial p} G(\hat{p})-\frac{\partial d(\tilde{p})}{\partial p} G(\tilde{p})\right\}(\hat{p}-\tilde{p})<0
$$


which contradicts (A.12) since $\hat{p} \neq \tilde{p}$.

Proposition 1 is proved by Lemmas A.1, A.2, A.3 and A.4.

\section{Proof of Lemma 4}

Proof. We first note that the unconstrained maximum occurs either at the maximizer of $R_{i}^{d}$ or at the price $\bar{p}_{i}$ such that $v_{i}\left(\bar{p}_{i}\right)=d_{i}\left(\bar{p}_{i}, p_{-i}\right)$. Let us consider the demand function $d_{i}\left(p_{i}, p_{-i}\right)=c_{i}-b_{i i} p_{i}$ where $c_{i}=a_{i}+\sum_{j=1, j \neq i}^{n} b_{i j} p_{j}$ for all $i=1, \ldots, n$. It is obvious that

$$
p_{i}^{\max }=\frac{c_{i}}{b_{i i}}>0
$$

Then the revenue defined by demand, $R_{i}^{d}$, becomes

$$
\begin{aligned}
R_{i}^{d}\left(p_{i}, p_{-i}\right)=\left(p_{i}-q_{i}\right)\left(c_{i}-b_{i i} p_{i}\right)+q_{i} \mu_{i} & \\
& \quad-\left(h_{i}-q_{i}\right)\left(c_{i}-b_{i i} p_{i}\right) F_{i}\left(c_{i}-b_{i i} p_{i}\right)+\left(h_{i}-q_{i}\right) \int_{0}^{c_{i}-b_{i i} p_{i}} x f(x) d x
\end{aligned}
$$

and its unconstrained maximizer can be obtained by considering

$$
\frac{\partial R_{i}^{d}}{\partial p_{i}}=c_{i}+b_{i i} q_{i}-2 b_{i i} p_{i}+b_{i i}\left(h_{i}-q_{i}\right) F\left(c_{i}-b_{i i} p_{i}\right)=0
$$

From the strictly pseudoconcavity of $R^{d}$, we know that before the maximum the first-order derivative is positive and after the maximum it is negative. By (A.15) into (A.17), we obtain

$$
\begin{aligned}
c_{i}+b_{i i} q_{i}-2 b_{i i} \frac{c_{i}}{b_{i i}}+b_{i i}\left(h_{i}-q_{i}\right) F(0) & =-c_{i}+b_{i i} q_{i} \\
& =-d_{i}\left(q_{i}, p_{-i}\right) \\
& <0
\end{aligned}
$$

Therefore we obtain

$$
p_{i, \text { unconstrained }}^{d, *}<p_{i}^{\max }
$$

The price $\bar{p}_{i}$ can be obtained by considering

$$
F^{-1}\left(\frac{p_{i}-q_{i}}{h_{i}-q_{i}}\right)=c_{i}-b_{i i} p_{i}
$$

We observe that at $p_{i}^{\max }=\frac{c_{i}}{b_{i i}}$, we have

$$
F^{-1}\left(\frac{p_{i}^{\max }-q_{i}}{h_{i}-q_{i}}\right)>c_{i}-b_{i i} p_{i}^{\max }=0
$$


Since $v_{i}$ is increasing and $d_{i}$ is decreasing, we obtain

$$
\bar{p}_{i}<p_{i}^{\max }
$$

From (A.18) and (A.19), we obtain the desired result (25).

\section{Proof of Lemma 5}

Proof. From Lemma 1, we have

$$
q_{i}=p_{i}^{*} \geq \bar{p}_{i} \geq q_{i}
$$

Hence, we obtain

$$
p_{i}^{*}=q_{i}=\bar{p}_{i}
$$

Suppose $\left.\frac{\partial R_{i}^{d}\left(p_{i}, p_{-i}^{*}\right)}{\partial p_{i}}\right|_{p_{i}=q_{i}}>0$. Since $R_{i}^{d}\left(p_{i}, p_{-i}^{*}\right)$ is strictly pseudoconcave, there exists $\tilde{p}_{i}>q_{i}$ (and $\left.\tilde{p}_{i} \leq h_{i}\right)$, such that $R_{i}^{d}\left(\tilde{p}_{i}, p_{-i}^{*}\right)>R_{i}^{d}\left(p_{i}^{*}, p_{-i}^{*}\right)$. Therefore, we must have

$$
\left.\frac{\partial R_{i}^{d}\left(p_{i}, p_{-i}^{*}\right)}{\partial p_{i}}\right|_{p_{i}=q_{i}} \leq 0
$$

According to equation (A.2), we have

$$
\frac{\partial R_{i}^{v}\left(p_{i}, p_{-i}^{*}\right)}{\partial p_{i}}=v\left(p_{i}\right) \geq 0 \quad \text { for all } \quad p_{i} \geq q_{i}
$$

From equation (A.4) and (A.22), at $p_{i}=\bar{p}_{i}$, we have

$$
\frac{\partial R_{i}^{v}\left(p_{i}, p_{-i}^{*}\right)}{\partial p_{i}}=v\left(\bar{p}_{i}\right)=d_{i}\left(\bar{p}_{i}\right)=\frac{\partial R_{i}^{d}\left(p_{i}, p_{-i}^{*}\right)}{\partial p_{i}} \geq 0
$$

If $v\left(q_{i}\right)>0$, we obtain

$$
\left.\frac{\partial R_{i}^{d}\left(p_{i}, p_{-i}^{*}\right)}{\partial p_{i}}\right|_{p_{i}=q_{i}}=d_{i}\left(\bar{q}_{i}\right)=v\left(\bar{q}_{i}\right)>0
$$

which contradicts equation (A.21). Hence we proved the lemma.

\section{Proof of Lemma 6}

Proof. First, suppose $\left.\frac{\partial R_{i}^{d}\left(p_{i}, p_{-i}^{*}\right)}{\partial p_{i}}\right|_{p_{i}=h_{i}}<0$. Since $R_{i}^{d}\left(p_{i}, p_{-i}^{*}\right)$ is strictly pseudoconcave, there exists $\hat{p}_{i}<h_{i}\left(\right.$ and $\left.\hat{p}_{i} \geq q_{i}\right)$, such that $R_{i}^{d}\left(\hat{p}_{i}, p_{-i}^{*}\right)>R_{i}^{d}\left(p_{i}^{*}, p_{-i}^{*}\right)$. Contradiction. 